Annuaire suisse de politique de développement

8 | 1988

Annuaire Suisse - Tiers Monde 1989

\title{
La coopération au développement entre la Suisse et l'Inde
}

Serge Chappatte et Kurt Vögele

\section{OpenEdition}

Édition électronique

URL : http://journals.openedition.org/aspd/1277

DOI : $10.4000 /$ aspd. 1277

ISSN : 1663-9669

Éditeur

Institut de hautes études internationales et du développement

\section{Édition imprimée}

Date de publication : 1 décembre 1988

Pagination : 157-166

ISSN : 1660-5934

\section{Référence électronique}

Serge Chappatte et Kurt Vögele, "La coopération au développement entre la Suisse et I'Inde »,

Annuaire suisse de politique de développement [En ligne], 8| 1988, mis en ligne le 13 mars 2013, consulté le 08 septembre 2020. URL : http://journals.openedition.org/aspd/1277 ; DOI : https:// doi.org/10.4000/aspd. 1277 


\title{
La coopération au développement entre la Suisse et l'Inde
}

\author{
Serge Chappatte et Kurt Vögele,
}

\section{Place de l'Inde dans la coopération suisse au développement}

De par la taille de sa population, de par son rôle politique, l'Inde ne peut être comparée à aucun des autres pays du Tiers Monde dans lequel la DDA concentre ses activités. Continent dans un continent, l'Inde occupe une surface égale à celle de l'Europe occidentale.

L'Inde est le principal pays bénéficiaire de la coopération suisse au développement; au cours de ces cinq dernières années, le volume annuel moyen des versements de la Direction de la coopération au développement et de l'aide humanitaire s'est monté à 35 millions de francs. L'Inde fut aussi le premier pays de concentration de la DDA à être doté d'un bureau de coordination, à la fois antenne et bras étendu de la DDA dans ses principaux pays d'activités. Ce fut en 1968 déjà. L'extension du programme dans le pays, en particulier dans le sud, la complexité des actions entreprises conduisirent la DDA à ouvrir en 1986 un sous-bureau de coordination à Bangalore.

\begin{tabular}{|c|c|c|c|}
\hline \multirow[t]{2}{*}{ Années } & \multicolumn{2}{|c|}{ APD } & \multirow{2}{*}{$\begin{array}{l}\text { Aide des } \\
\text { ONGs suisses }\end{array}$} \\
\hline & dons & prêts & \\
\hline 1965 & 1.9 & 1.9 & 3.8 \\
\hline 1970 & 6.3 & 6.3 & 9.0 \\
\hline 1975 & 7.4 & 29.8 & 11.5 \\
\hline 1980 & 27.7 & 0.1 & 14.4 \\
\hline 1981 & 10.3 & 0.4 & 13.7 \\
\hline 1982 & 37.5 & $\because-$ & 14.3 \\
\hline 1983 & 45.6 &.- & 14.5 \\
\hline 1984 & 48.2 &.- & 15.5 \\
\hline 1985 & 31.8 & 2.7 & 17.1 \\
\hline 1986 & 16.1 & 2.2 & 17.0 \\
\hline 1987 & 33.1 & 2.0 & 15.4 \\
\hline
\end{tabular}


Les grandes étapes de l'activité de la DDA en Inde peuvent se résumer comme suit:

- Créée en 1961, la DDA lance ses deux premiers projets bilatéraux au cours de 1963; ces deux projets, celui de l'élevage au Kerala et l'action en faveur des réfugiés tibétains occupent une place importante dans l'arbre généalogique de la DDA en Inde car ils ont tous deux influencé directement ou indirectement toute une série de programmes et de projets en réalisation.

Déjà avant 1961, de nombreuses oeuvres d'entraide privées suisses, confessionelles avant tout, étaient actives en Inde, parfois depuis de nombreuses années; la DDA put capitaliser l'expérience gagnée par ces organisations.

- La motion du conseiller national Kloter déposée à la suite de l'essai nucléaire effectué par l'Inde contraignit la DDA à ne démarrer aucun nouveau projet dans le pays durant la période 1974-1976;

- La fin des années 1970 marque le début d'un certain nombre de projets de formation professionnelle, en électronique, en meunerie, etc. Des projets virent également le jour dans la recherche appliquée au niveau universitaire, en biochimie et en énergie solaire;

- Au début des années 1980, démarrèrent dans plusieurs secteurs des projets qui occupent maintenant une place déterminante dans le programme de la DDA, à savoir la sériciculture, le crédit rural, la collaboration avec des organisations non gouvernementales indiennes et la formation professionnelle; ces projets sont basés sur des années de coopération technique patiente dans les mêmes secteurs et à travers lesquels des expériences suffisamment probantes ont pu être gagnées pour justifier des actions de grand style;

- L'action de la DDA vise toujours davantage une collaboration plus étroite entre le gouvernement indien et les oeuvres d'entraide privées indiennes dans le but d'accroître l'efficacité sociale "à la base" des programmes gouvernementaux et d'encourager la compréhension des planificateurs pour les problèmes de réalisation et les besoins d'un développement orienté socialement.

\section{Le programme de coopération au développement de la Suisse en Inde}

Depuis son indépendance, l'Inde a enregistré des progrès importants non seulement au niveau économique et social mais également dans la planification de son développement et dans les stratégies d'allocation de ressources. Le développement spectaculaire de l'agriculture, le renforcement de l'industrie, le niveau relativement bas de la dette, la gestion budgétaire et financière saine, sont autant d'éléments qui ont permis à l'Inde de connaître de bons taux de croissance stables et de dégager finalement suffisamment de ressources pour un programme de lutte contre la pauvreté. L'Inde est certainement un des rares 
pays du Tiers Monde à avoir une politique, une stratégie et des ressources macro-économiques importantes de lutte contre la pauvreté. Certes, dans de nombreux secteurs, des problèmes importants restent à résoudre (le développement de l'agriculture à surtout bénéficié à certaines céréales, certaines régions et certaines classes de la population rurale; le développement industriel est parfois anarchique et conduit à la pollution, les programmes de pauvreté sont loin d'être efficaces par la primauté donnée à la planification des besoins plutôt qu'à la réalisation sur le terrain, etc.), mais ils ont été en quelque sorte défrichés par la planification indienne.

Une conclusion importante s'impose dès lors pour le travail de la coopération suisse au développement en Inde: il n'y a pas en Inde de secteur grossièrement négligé par la planification nationale, il n'y a que des lacunes de qualité que les partenaires au développement de l'Inde peuvent aider à combler.

Si la DDA ne veut pas se borner en Inde à ne financer simplement que des programmes existants et préparés par l'administration, mais bien envisager un véritable dialogue avec l'Inde, les points suivants doivent être pris en considération:

- Les contributions relativement modestes de notre programmes doivent être engagées au niveau de petits projets, lorsque la qualité des programmes de développement doit être améliorée;

- Il peut durer parfois des années jusqu'à ce que nos partenaires et nous soyons tombés d'accord sur l'existence d'une niche de qualité;

- Le choix de nos projets doit être guidé à l'intérieur de nos grands secteurs de concentration par un certain pragmatisme;

- Le choix de nos partenaires doit être conduit par la motivation et le fait de partager une perspective commune.

La coopération suisse au développement en Inde a donc fait preuve de beaucoup de pragmatisme dans le choix des projets, parfois de manière purement fortuite, parfois à la suite de constellations d'intérêts particuliers. Les activités du programme actuel ont malgré tout une forte logique interne et se caractérisent par une concentration sectorielle marquée.

Les grands secteurs dans lesquels la coopération suisse au développement avec l'Inde est active à l'heure actuelle sont les suivants:

a) Amélioration de l'utilisation du sol dans des zones écologiquement menacées (zones sèches et montagneuses): une grande partie de la population rurale de l'Inde dépend de l'agriculture en zone sèche et est restée à l'écart des résultats remarquables de la révolution verte. Le plateau du Deccan, la zone des collines des Ghats, qui bordent l'Inde vers l'Ouest, et l'Himalaya appartiennent à cette catégorie. Des projets ont été réalisés ou sont envisagés avec les autorités gouvernementales mais aussi avec des organisations volontaires indiennes. 
b) Elevage, fourrage et économie laitière: il s'agit là des projets reliés plus ou moins directement au projet pionnier du Kerala, qu'il s'agisse de projets d'élevage comme au Rajasthan et en Andhra Pradesh et peut-être aussi en Orissa, de formation de cadres pour les coopératives laitières ou d'appui à l'organisation de la collecte de la transformation et de la commercialisation de lait sur une base coopérative.

c) Petite industrie et artisanat rural: une série d'actions plus ou moins dispersées ont été réalisées au fil des années; ce n'est vraiment qu'avec le début de la collaboration dans le domaine de la sériciculture que ce secteur de la promotion de l'emploi rural non agricole a pris une certaine importance dans le programme de la DDA. Les actions dans le secteur de la sériciculture ont été nombreuses et culminent dans la préparation d'un programme important réalisé avec le Gouvernement indien et la Banque mondiale. La collaboration avec la Banque nationale pour le développement de l'agriculture et du développement rural (NABARD) s'oriente maintenant nettement vers le secteur rural non agricole avec l'ambition d'y provoquer une stratégie encore déficiente de liaison entre le crédit et les mesures techniques d'appui, la formation, etc.

d) Formation professionnelle et des cadres: démarrées au milieu des années septante, les actions entreprises en matière de développement de la formation professionnelle et de cadres ont été fortement intensifiées au cours des années quatre-vingt et offrent toutes les chances d'un développement substantiel dans les dix années à venir. Les domaines de collaboration ont été la mécanique, l'outillage, l'électronique, l'électromécanique, la meunerie. La formation de cadres pour le développement rural a suivi logiquement la longue implication de la DDA dans le secteur de l'élevage.

e) Mesures d'appui et activités complémentaires: dans cette catégorie sont regroupées des mesures spécifiques dans les domaines du crédit, de la recherche et du développement communautaire qui permettent de mieux atteindre les buts qui ont été fixés dans les quatre grandes familles de projet sus-mentionnées. Le secteur du crédit a connu un développement spectaculaire depuis le début de la collaboration à la fin des années septante avec la NABARD. La recherche s'est concentrée depuis la fin des années septante sur la biochimie et la biotechnologie. Le développement de structures propices au développement en zones rurales a été encouragé à travers les actions de développement communautaire des oeuvres d'entraide privées indiennes avec lesquelles la DDA collabore.

III

Dans le domaine géographique, nos efforts de concentration n'ont pas rencontré autant de succès. Une des raisons en est que le Gouvernement indien désire ne favoriser aucune région particulière de l'Inde et refuse officiellement toute concentration géographique. Une certaine concentration ne s'en est pas moins dégagée automatiquement. Dans un pays-continent comme l'Inde, notre 
expérience a montré que des années de collaboration sont nécessaires jusqu'à ce que des contacts approfondis aient été noués au niveau des Etats de l'Union indienne et qu'une base de confiance soit développée qui permette la réalisation harmonieuse de projets et de programmes.

Notre programme de collaboration bilatérale officielle avec le Gouvernement montre une certaine concentration dans les Etats du Sud du pays, alors que notre programme de coopération avec des organisations non gouvernementales indiennes est plutôt ancré dans la plaine gangétique du Nord de l'Inde.

\section{IV}

Nous avons déjà brièvement esquissé ci-dessus quelques-unes des réflexions stratégiques qui doivent guider l'action d'un petit partenaire au développement de l'Inde. Nous ne reviendrons pas sur la concentration qui est absolument nécessaire pour éviter de devenir totalement insignifiants dans cet océan. Mais comment assurer à la coopération suisse au développement un impact optimum? II faut constater qu'en Inde aucun partenaire bilatéral ne peut avoir une influence déterminante au niveau central sur les décisions en matière de politique économique, sur les politiques d'ajustement, etc. Notre expérience montre également que des processus de prise de conscience, que le lancement de programmes novateurs sont déclenchés souvent dans des activités pratiques et souvent modestes au niveau du terrain. De cette manière, la Suisse peut jouer un rôle écouté dans le dialogue des politiques en montrant des alternatives ou des compléments sociaux et techniques efficaces aux politiques en place, en se lançant dans des activités novatrices risquées, mais porteuses d'espoirs et de solutions mieux adaptées tout en étant macro-économiquement valables. C'est ce qui s'est passé dans certains secteurs comme l'élevage, où les projets de la famille du Kerala ont permis de dégager une politique d'élevage appropriée et efficace, dans le secteur du fourrage, par l'accent mis sur la complémentarité de ces activités avec l'élevage; en formation professionnelle, par une plus grande relation à la pratique et aux réalités de la production; dans le domaine du crédit rural où, dans certains districts de l'Inde, des solutions originales ont pu être suggérées pour accroître l'efficacité du crédit comme moteur de développement, etc. D'autres exemples de ce genre pourraient être cités. Ce rôle n'est cependant pas facile à jouer. II nécessite une forte débauche d'énergie. Le problème du développement de l'Inde, vu sous l'angle de la coopération internationale au développement, n'est pas d'abord un problème de finances.

\section{Les traits de la coopération au développement avec l'Inde}

L'Inde finance elle-même près de $95 \%$ de son effort de développement. Les quelque 5 milliards de dollars par année qui constituent les $5 \%$ restants sont 
couverts pour près de la moitié par la Banque mondiale. Lors de la dernière réunion du "Consortium de l'Inde" de la Banque mondiale, en juin 1988 à Paris, les pays membres, dont la Suisse, ont indiqué qu'ils étaient prêts à consentir pour l'année à venir des engagements d'un montant de 6,3 milliards de dollars dont 3,9 milliards à titre concessionnel. La part de la Suisse est d'environ 0,5\% du total du Consortium.

Et pourtant l'Inde ne saurait se passer sans mal de l'aide au développement. Ce paradoxe n'en est un qu'en surface. Technologiquement auto-suffisante dans de nombreux domaines, l'Inde connaît cependant quelques insuffisances pour les domaines de la haute technologie par exemple, où le recours à des biens d'équipement étrangers et à de nouvelles technologies et savoir-faire est indispensable. La communauté internationale marque généralement son appréciation de l'effort entrepris par l'Inde, comme en témoignent les montants d'aide annoncés chaque année par les pays-membres de la réunion du Consortium de I'Inde, mais regrette que celui-ci tarde à déployer des effets positifs pour la masse des pauvres, en particulier dans les régions rurales. La place de la lutte contre la pauvreté, pierre angulaire de nombreux programmes bilatéraux d'aide au développement, dans les programmes officiels de développement, a toujours été et restera une pomme de discorde.

\section{II}

L'appareil de l'Etat a, en tout cas au niveau conceptuel, créé des conditions qui font de l'Inde un des pays les plus adéquats pour les critères de la coopération suisse au développement, en particulier sa volonté de s'aider soi-même. L'Inde est consciente du problème de la pauvreté; la démocratie indienne offre de bonnes chances de développement d'initiatives propres. Le plan quinquennal actuel souligne, par exemple, de manière claire l'importance du volontariat et des efforts privés dans le processus de développement du pays. Ces phénomènes sont étroitement liés à un sentiment de confiance en soi, soulignent le développement indépendant du pays et rappellent ses sources riches et profondes. Cette sûreté de soi conduit souvent à l'enthousiasme, à l'intérêt pour ce qui est nouveau.

La médaille a certainement aussi son revers, comme le montre l'expérience de la DDA qui a dû et doit tenir compte de certaines caractéristiques propres à l'Inde:

- Le savoir et le faire sont parfois totalement séparés l'un de l'autre. Les travaux intellectuels (planification par exemple) comptent plus que l'exécution. La formation professionnelle est presque toujours théorique et peu pratique.

- Les structures de gestion sont en général plutôt interventionnistes et autoritaires; la délégation des compétences est rarement pratiquée. Cela peut conduire à une bureaucratisation excessive et à une réglementation de nombreux domaines de la vie publique.

- La réalisation de buts quantitatifs de croissance est plus importante que les objectifs qualitatifs et socio-économiques.

- Les mesures ponctuelles ont la priorité sur une approche systémique. 
III

Espoirs et limites de la coopération suisse au développement

Dans quelle mesure a-t-il été possible à la DDA d'atteindre les deux principaux objectifs de la loi fédérale sur la coopération internationale au développement, à savoir le soutien de populations défavorisées dans les régions les plus pauvres du pays et l'appui à un développement autonome et indépendant du pays ?

S'il fallait répondre de manière étroite au premier critère, il faudrait dire qu'il est difficile d'atteindre directement les plus pauvres du pays et à les impliquer dans un programme de coopération; mais est-ce vraiment toujours nécessaire? L'influence indirecte est aussi efficace et nous constatons qu'à travers sa collaboration, la DDA a effectué une contribution importante en faveur des couches de populations les plus pauvres du pays. Notre collaboration a plutôt contribué à réduire le dualisme économique en donnant une assise plus vaste socialement aux programmes de développement, en soignant le détail de l'exécution de projets, en renforçant les institutions qui, elles, atteignent directement les plus pauvres, en soutenant l'effort de croissance du pays, qui seul, peut déboucher sur une stratégie de lutte contre les causes de la pauvreté, etc.

Quant à l'objectif de renforcer la capacité d'assumer son propre développement, à tous les niveaux, il faut reconnaître que l'action de la DDA a très souvent permis de l'atteindre. Cela est facilité par le fait que la politique économique du pays a fait sienne la maxime du développement autonome et que le pays a les moyens de l'imposer dans le contexte international.

IV

II est impossible, voire inapproprié de concentrer toutes nos activités de manière unidimensionnelle sur la lutte directe contre la pauvreté. L'analyse des expériences de la DDA montre plutôt qu'il faut continuer à participer en Inde de manière sélective à des efforts de développement du gouvernement qu'ils soient partie intégrante ou non de la lutte directe contre la pauvreté, à condition qu'ils :

a) s'orientent aux besoins et aux possibilités des bénéficiaires,

b) contribuent à mettre sur pied des structures économiques et sociales qui permettent de poursuivre la lutte contre la perpétuation de la pauvreté sous toutes ses formes,

c) encouragent les initiatives et la volonté d'entreprendre un développement autonome,

d) offrent des possibilités d'engager de manière efficace les montants relativement modestes de la coopération suisse au développement.

\section{V}

La collaboration avec les organisations indiennes non gouvernementales a déjà été suffisamment intensive pour nous permettre de tirer deux conclusions principales, à savoir: 
- Les organisations volontaires indiennes méritent d'être soutenues dans leurs efforts de trouver des alternatives économiques et sociales viables aux programmes bureaucratiques du gouvernement. II serait contre-productif de s'associer à des engagements qui mènent à une duplication des programmes gouvernementaux ou encouragent la création de bureaucraties parallèles dans le secteur non gouvernemental;

- Les organisations non gouvernementales méritent d'êtres encouragées dans leurs efforts d'associer des groupes bénéficiaires à la planification et à l'exécution de programmes gouvernementaux qui sont généralement bien conçus mais pèchent au niveau de l'exécution. C'est le cas de la création d'emplois en zones rurales, du développement des services de santé, du crédit, du soutien aux petits paysans et aux paysans marginaux, des programmes d'appui à l'agriculture en zones sèches, etc.

\section{VI}

Le rôle de la coopération suisse au développement devrait être de créer des ponts entre l'administration gouvernementale et la population, que les organisations non gouvernementales représentent de manière souvent adéquate. La DDA s'efforce également de renforcer l'efficacité des organisations volontaires comme porte-parole de la population par des contributions au renforcement institutionnel de ces organisations dans les domaines de l'organisation, de la gestion, et de la compétence technique.

C'est également dans ce même état d'esprit que la DDA soutient les efforts des oeuvres d'entraide privées suisses actives en Inde tout en respectant l'autonomie de ces organisations et leurs priorités spécifiques en matière de politique de développement.

\section{VII}

La planification des projets de développement n'est parfois qu'indirectement influencée par la pratique sur le terrain; les planificateurs partent en général de réflexions macro-économiques et donnent la préférence à des objectifs quantitatifs. La planification au niveau micro-économique et les aspects qualitatifs jouent un rôle relativement secondaire; les interventions de politiques de développement sont généralement limitées à un catalogue de mesures planifiées au niveau général et dotées de budgets impressionnants. Les processus de décision sont centralisés et structurés de manière extrêmement hiérarchique.

L'exécution des mesures de développement se base sur un nombre considérable de directives et de règles et partent de la conviction qu'un volume financier suffisant et un nombre adéquat de fonctionnaires permettront d'atteindre plus ou moins automatiquement les buts fixés.

La motivation et l'esprit d'innovation des responsables de l'exécution ne sont pas toujours stimulés; ceux-ci sont avant tout des exécutants et on n'attend pas d'eux qu'ils développent leur propre initiative. 
Le développement n'est pas compris comme le fruit d'une collaboration entre la population et l'appareil étatique, mais comme un bien que le gouvernement administre et distribue de manière plus ou moins efficace.

Les rapports de force aux différents échelons de la structure gouvernementale peuvent aussi être utilisés pour l'enrichissement personnel; les bénéficiaires sont privés d'une partie des prestations de développement qui sont prévues pour eux. Le "feed-back» manque. Les expériences positives et négatives ont de la peine à refaire leur chemin jusqu'aux personnes qui ont formulé les concepts de départ. L'évaluation, dans le sens critique mais constructif qu'elle doit avoir, n'est pas toujours du goût des responsables indiens. La capacité d'apprentissage est limitée, cela d'autant plus que les planificateurs se meuvent dans l'ambiance confortable qu'ils ont créée loin des problèmes du terrain.

\section{VIII}

La collaboration avec les organisations non gouvernementales indiennes a commencé en 1980 dans le but d'avoir un contact plus direct avec la population. Les organisations volontaires indiennes ont une grande réputation en la matière. Les premières expériences furent encourageantes, mais force est de reconnaître qu'il faudra encore de nombreux efforts pour que les buts envisagés soient atteints. Un développement trop rapide de la collaboration avec les organisations volontaires indiennes peut finalement nuire de manière irréversible à ces organisations. La communauté internationale d'aide, qui a de plus en plus tendance à utiliser le canal ONG, est placée devant une responsabilité extrêmement grande.

La collaboration avec ces organisations devrait porter aussi sur l'amélioration de leur fonctionnement, en particulier en ce qui concerne l'organisation du travail, la préparation, le suivi et l'évaluation critique de leurs propres activités, les compétences techniques et sociales, la formation de leurs cadres, etc.

\section{Regard vers l'avenir en guise de conclusion}

Les efforts du gouvernement indien en matière de développement ont connu certains succès dont ne peuvent pas se vanter beaucoup d'autres pays du Tiers Monde. La sécheresse de 1987, pourtant une des plus importantes à avoir jamais frappé le pays, a pu être combattue et ses conséquences réduites par les forces mêmes du pays, pratiquement sans aucun appui extérieur. D'importateur chronique de céréales, l'Inde est arrivée à une situation que d'aucuns lui envient d'avoir des réserves qui lui permettent de faire face aux coups durs, voire dans certains cas, peut-être symboliques encore, de venir en aide à ses voisins.

Mais les défis qui lui restent posés sont incroyablement graves. La cohésion nationale est fortement secouée par des tendances sécessionnistes, le fossé entre riches et pauvres exacerbe les rivalités socio-économiques et met en 
cause la texture même de la société et de la démocratie indienne. Il y a à l'heure actuelle davantage de pauvres en Inde que dans l'ensemble de l'Afrique subsaharienne.

Face à cette situation, les objectifs de la coopération suisse au développement avec l'Inde ne peuvent pas être très différents de ce qu'il ont été jusqu'à présent. L'importance capitale et centrale reste le problème de la pauvreté; toutes les actions possibles doivent être examinées à l'aune de leur incidence, directe ou indirecte, sur la pauvreté, et cela d'autant plus que, pratiquement, le travail de la DDA ne peut pas toujours se dérouler en contact direct avec les plus pauvres du pays. La contribution de la Suisse à la résorption de la pauvreté passe par des mesures à différents niveaux; la négociation d'un important accord commercial international peut finalement avoir plus d'effets sur la réduction de la pauvreté qu'une action ponctuelle au niveau d'un ou deux villages. 